\title{
Whispering Gallery Mode Based Optical Fiber Sensor for Measuring Concentration of Salt Solution
}

\author{
Chia-Chin Chiang and Jian-Cin Chao \\ Department of Mechanical Engineering, National Kaohsiung University of Applied Sciences, 415 Chien-Kung Road, \\ Kaohsiung 807, Taiwan
}

Correspondence should be addressed to Chia-Chin Chiang; ccchiang@cc.kuas.edu.tw

Received 14 September 2013; Accepted 18 October 2013

Academic Editor: Liang-Wen Ji

Copyright (C) 2013 C.-C. Chiang and J.-C. Chao. This is an open access article distributed under the Creative Commons Attribution License, which permits unrestricted use, distribution, and reproduction in any medium, provided the original work is properly cited.

\begin{abstract}
An optical fiber solution-concentration sensor based on whispering gallery mode (WGM) is proposed in this paper. The WGM solution-concentration sensors were used to measure salt solutions, in which the concentrations ranged from $1 \%$ to $25 \%$ and the wavelength drifted from the left to the right. The experimental results showed an average sensitivity of approximately $0.372 \mathrm{~nm} / \%$ and an $R^{2}$ linearity of 0.8835 . The proposed WGM sensors are of low cost, feasible for mass production, and durable for solutionconcentration sensing.
\end{abstract}

\section{Introduction}

The whispering gallery mode (WGM), also known as whispering gallery wave, was first discovered and proposed in 1912 by the British physicist John William Strutt (Raleigh) [1], who studied the propagation of sound along wall curvatures. The macrobending optical fiber induces a WGM. When light travels via WGMs from the fundamental mode of fibers to bends, the coupling between the core mode and the cladding mode facilitates the propagation.

In 1990, Morgan et al. [2] introduced a fiber-optic WGM sensor that was applicable to various angles of fiber bends. They discovered that smaller bend diameters and larger bend angles produced more distinct interference curves. This effect results from the different wavelengths that are produced by different fiber bend angles. Variations in wavelengths can be used to create fiber-optic WGM sensors with various wavelengths.

In 2002, the U-shaped fiber-optic $\mathrm{pH}$ of sensors based on evanescent wave absorption was reported by Gupta and Sharma [3]. They discovered that the sensitivity of the sensor increases with the decrease in the bending radius of the $\mathrm{U}$ shaped fiber sensor.

In 2009, Wang et al. $[4,5]$ presented a bending interferometric fiber-optic sensor. They connected two photodiodes to both sides of a microbending fiber-based sensor to measure the output power. In addition, they used the displacement of a single-process micromotion platform to measure bend loss. Bend radii in optical fibers were changed to alter the wavelengths. Their experimental results demonstrated that a bend diameter of $18 \mathrm{~mm}$ produced insufficient interference at the bends, whereas a bend diameter of less than $15 \mathrm{~mm}$ increased the risk of rupture.

In 2009, a WGM refractive index sensor was reported $[6,7]$, in which optical fibers were bent in the shape of a ring to form WGMs. The sensor is the measurement of refractive index from the different organic solutions. The sensor was bent to a diameter of $19.3 \mathrm{~mm}$, and the refractive index sensitivity is up to $725.76 \mathrm{~nm} / \mathrm{RIU}$ (refractive index units).

In 2010, a similar bending interferometric fiber-optic sensor was proposed [8]. A taper in the center of an optical fiber was formed, and a sensing optical fiber was placed next to the taper. An adjustable laser was transmitted from a single-mode optical fiber through the taper to the sensing optical fiber. The laser was then totally reflected, coupled, and transmitted back to the single-mode optical fiber. Consequently, this principle served as the basis of the proposed bending interferometric fiber-optic sensor. This sensor has a sensitivity of $16.1 \mathrm{~nm} / \mathrm{RIU}$ to solutions. 


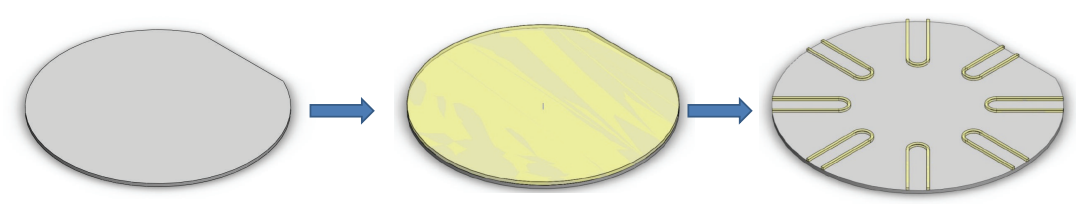

Clean the wafers

Apply SU-8 100 to the wafer

Exposure and development of the structure

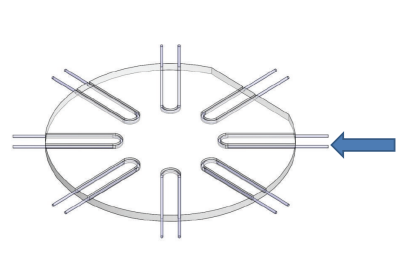

Insert the etched optical fibers

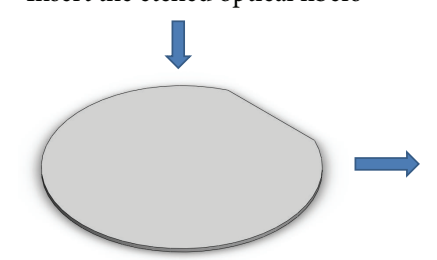

Wafer without a structure on top

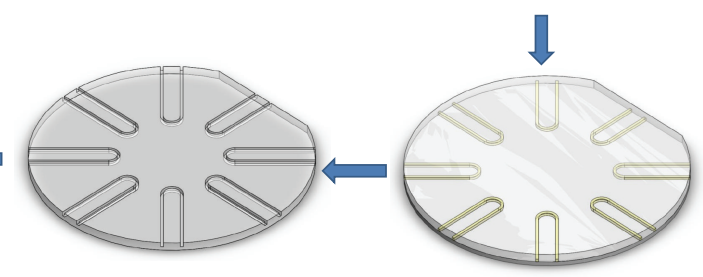

Delaminate the PDMS lower Apply PDMS layer

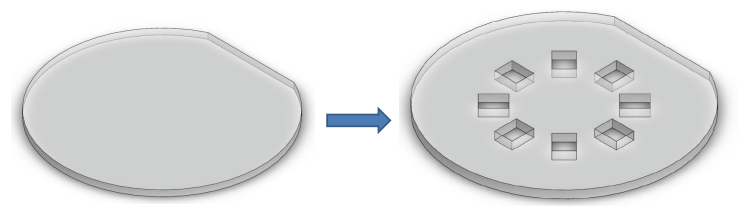

Apply a layer of PDMS and perform delamination

Open holes on PDMS (upper layer)

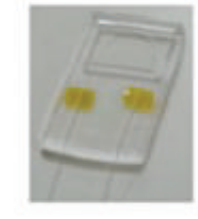

Image of a WGM sensor

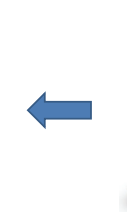

Competing the manufacturing
process of the WGM sensor

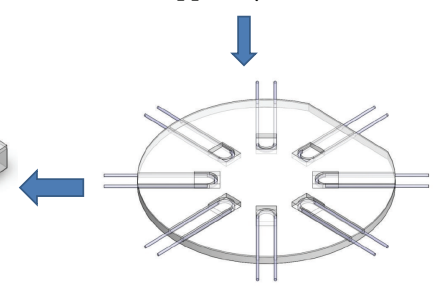

Connect the upper and lower layers using a corona treater

FIGURE 1: The manufacturing process of the WGM concentration sensor.

This study describes a macrobending-induced WGM fiber-optic sensor. Based on the analysis of the WGM spectrum, wavelength variations in optical fibers with differing bend radii were observed. The sensitivity and linearity of the sensors were also analyzed and calculated.

\section{Materials and Methods}

2.1. Process and Procedures of Optical Fiber Etching. The diameter of the optical fiber plays an important role in macrobending. As the fiber's diameter decreases, its flexibility increases. Therefore, the life of the bending fiber also increases. The optical fibers in this study were wet-etched using buffered oxide etch (BOE) to alter their diameters. A stripper was used to remove $3 \mathrm{~cm}$ of the external protective layer of the optical fiber. Fifty stripped optical fibers were adhered to a plastic holder. The holder with the fibers was placed inside a plastic box filled with BOE for etching. Fiber diameters were altered using various etching durations.

2.2. Optical Fiber Sensing Systems for Concentration Monitoring. This study successfully developed a WGM sensor which is of low cost, can be mass-produced, and has the ability to accurately control the bending radius of the optical fiber. Additionally, this process increases the accuracy of measuring differing concentration/refractive indices of liquids.

Figure 1 shows a flow chart of the manufacturing process of the proposed WGM concentration sensor. We used the replica molding method with polydimethylsiloxane (PDMS) to fabricate the WGM sensors. First, a microelectromechanical system (MEMS) process was used to produce the molds with an SU-8 100 structure. The base-layer PDMS was obtained by the replica molding method. Then, the upper and lower layers were connected using a corona treater to sandwich the U-bending optical fiber with two patterned PDMS layers. Finally, the sensor was fabricated using this process (Figure 1). Figure 2 shows the experimental setup of the concentration test. The WGM sensor was connected to a super luminescent diode (SLD) light source and an optical spectrum analyzer (OSA). Then, the WGM sensor was placed in salt solutions with differing concentrations. The spectra of the sensor were observed using the OSA. 


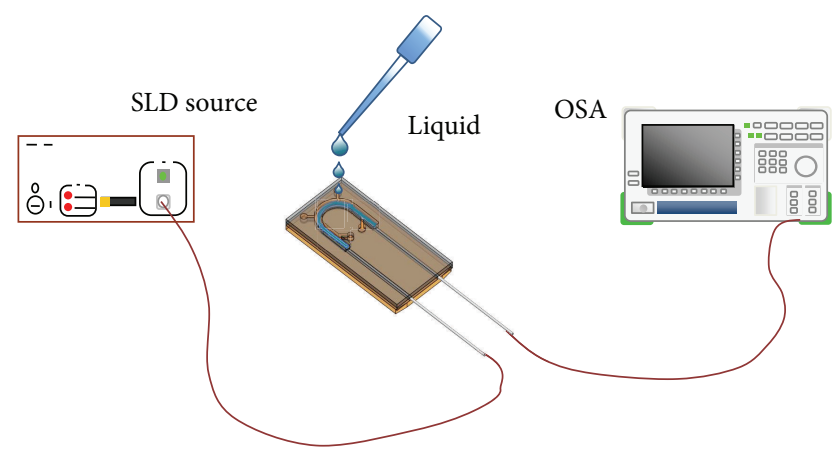

FIGURE 2: Experimental setup of the WGM solution-concentration sensing system.

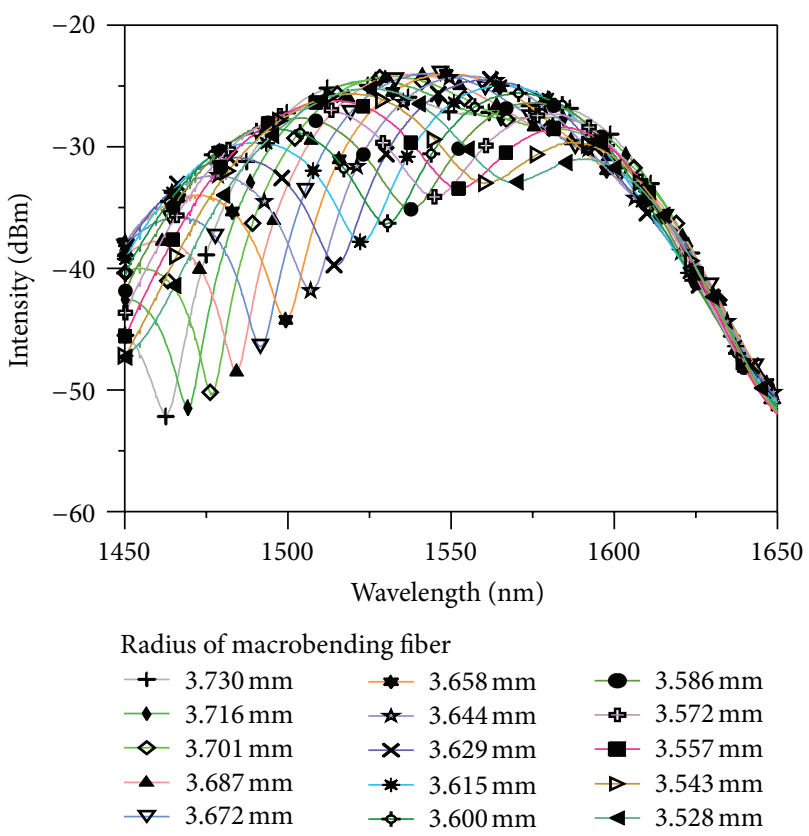

Figure 3: The spectra of the macrobending fiber with different bending radii.

\section{Results and Discussion}

The objective of this study is to macrobend the etched optical fiber to form a WGM character for sensing. The spectra of WGM concentration sensors were affected as the surrounding refractive index changed with different concentrations. Figure 3 shows the spectra of the macrobending fiber with different bending radii. The diameter of the optical fiber is $65 \mu \mathrm{m}$ with the fiber bending angle of $180^{\circ}$ as a U-shape. When the bending radius decreases, the wavelength of the WGM redshifts. When the bending radius is $3.629 \mathrm{~mm}$, the maximum interference loss is $39.693 \mathrm{dBm}$ and the wavelength position is $1514.1 \mathrm{~nm}$. The relationship between the WGM wavelength and the bend radius of the optical fiber is linear. As shown in Figure 4, the average slope is approximately $530.922 \mathrm{~nm} / \mathrm{mm}$ and $R^{2}$ linearity is 0.999 .

In the solution-concentration measurement experiment, the WGM spectra were observed to be closely related to the

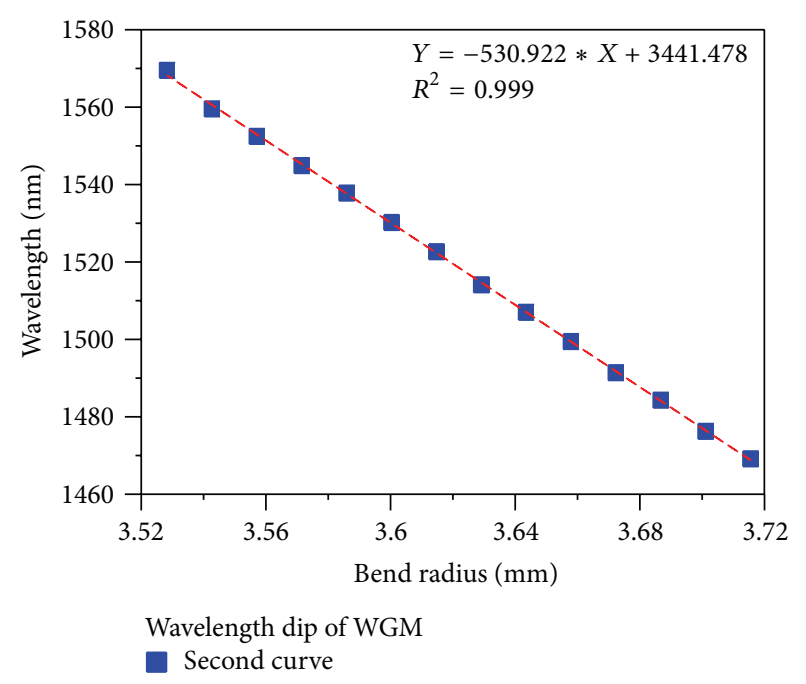

FIGURE 4: The relationship between the WGM wavelength and the bend radius of optical fiber.

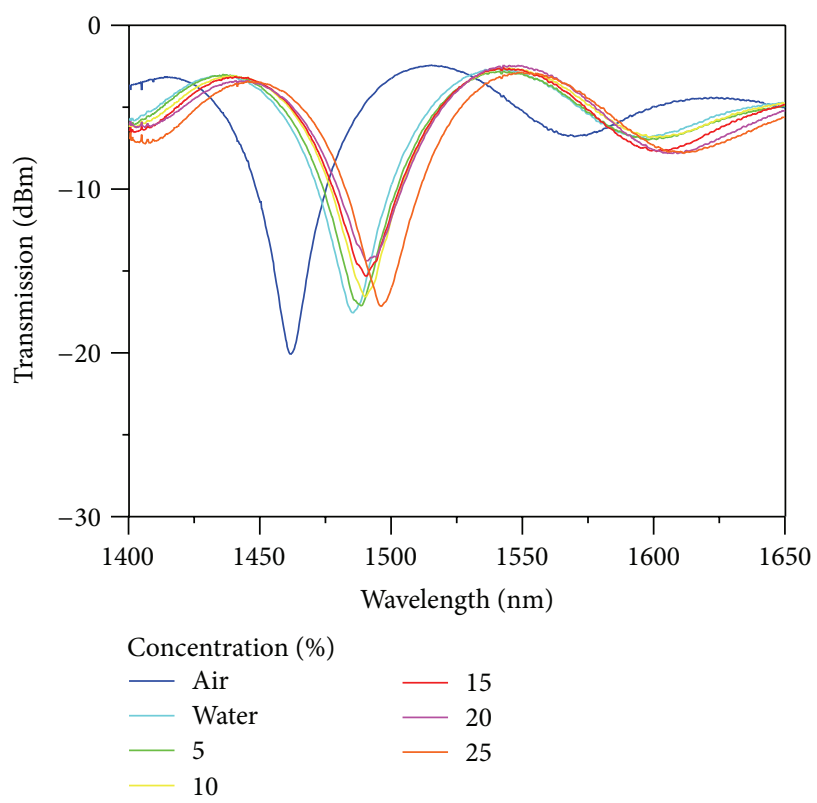

FIGURE 5: Interference spectra drifts with the concentration of salt solution.

concentration of the solution. This phenomenon shows that the difference between the refractive indices of two solution mediums affected the results of the WGM spectra. Figure 5 presents the spectrum drifts in salt solutions measured by the WGM concentration sensor. The resonant dip spectrum (in air) shows a significant interference loss, and the interference curves of the solutions with a concentration of $1-25 \%$ drifted toward the right gradually. In Figure 5, the wavelengths of the WGM sensor in air and water are $1461.63 \mathrm{~nm}$ and $1486 \mathrm{~nm}$, respectively. Owing to the significant change of refractive index between air and water, the wavelength shift is large (approximately $24.37 \mathrm{~nm}$ ).

Figure 6 shows the relationship between the concentration of salt solution and wavelength. The optical fibers with a 


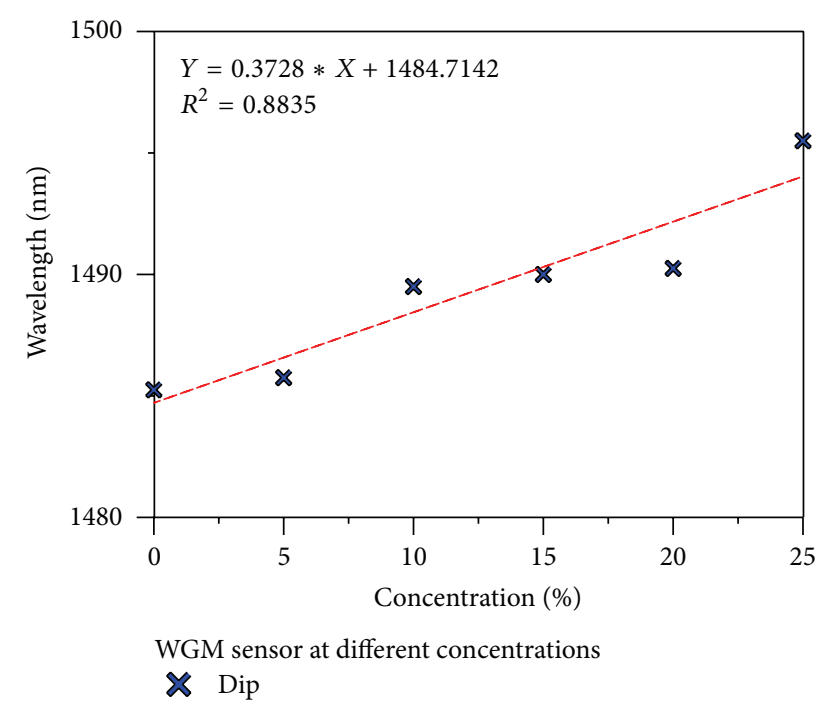

FIGURE 6: Relationship between the concentration of salt solution and wavelength.

diameter of $65 \mu \mathrm{m}$ and a bend radius of $3.5 \mathrm{~mm}$ were adopted to monitor salt solutions at a concentration of $1-25 \%$. The experimental results showed that the average sensitivity was $0.3728 \mathrm{~nm} / \%$ and $R^{2}$ linearity was 0.8835 .

The spectral analysis indicated that higher concentrations of salt solutions caused the resonant wavelength to drift toward the long wavelength side (red shift). On the contrary, when the media had a low refractive index, the interference spectra changed accordingly, and the resonant wavelength drifted toward the short wavelength side (blue shift). In short, the experiments proved the WGM concentration sensors to be extremely stable and highly reproducible.

\section{Conclusion}

This paper demonstrated the manufacturing process of the WGM concentration sensors. The results show the influences of refractive indices on the spectral characteristics of the fiber-optic WGM sensor. In addition, WGM solutionconcentration sensors were used to monitor the concentration of the salt solutions. The resonant dip spectrum (in air) shows a significant interference loss, and the interference curves of the solutions with a concentration of $1-25 \%$ drifted toward the right gradually. The results of salt-solution concentration monitoring showed an average sensitivity of $0.372 \mathrm{~nm} / \%$ and an $R^{2}$ linearity of 0.8835 . Additionally, the resonant wavelength shifted toward red as the concentration of salt solutions increased.

\section{Acknowledgment}

This work is supported by the National Science Council, Taiwan (Grant no. NSC 100-2628-E-151-002-MY3).

\section{References}

[1] L. Raleigh, "The problem of the whispering gallery," Scientific Papers, vol. 5, pp. 617-620, 1912.
[2] R. Morgan, J. S. Barton, P. G. Harper, and J. D. C. Jones, "Wavelength dependence of bending loss in monomode optical fibers: effect of the fiber buffer coating," Optics Letters, vol. 15, no. 17, pp. 947-949, 1990.

[3] B. D. Gupta and N. K. Sharma, "Fabrication and characterization of U-shaped fiber-optic $\mathrm{pH}$ probes," Sensors and Actuators $B$, vol. 82, no. 1, pp. 89-93, 2002.

[4] P. Wang, Y. Semenova, Q. Wu, and G. Farrell, "A macrobending fiber based micro-displacement sensor utilizing whisperinggallery modes," in 20th International Conference on Optical Fibre Sensors, vol. 7503 of Proceedings of the SPIE, October 2009, $75033 \mathrm{O}$.

[5] P. Wang, Y. Semenova, Q. Wu, and G. Farrell, "A macrobending fiber based micro-displacement sensor," in Proceedings of the International Symposium on Photonics and Optoelectronics (SOPO '10), pp. 1-4, June 2010.

[6] P. Wang, Y. Semenova, Q. Wu, G. Farrell, Y. Ti, and J. Zheng, "Macrobending single-mode fiber-based refractometer," Applied Optics, vol. 48, no. 31, pp. 6044-6049, 2009.

[7] P. Wang, Y. Semenova, Y. Li, Q. Wu, and G. Farrell, "A macrobending singlemode fiber refractive index sensor for low refractive index liquids," Photonics Letters of Poland, vol. 2, no. 2, pp. 67-69, 2010.

[8] A. Boleininger, T. Lake, S. Hami, and C. Vallance, "Whispering gallery modes in standard optical fibres for fibre profiling measurements and sensing of unlabelled chemical species," Sensors, vol. 10, no. 3, pp. 1765-1781, 2010. 

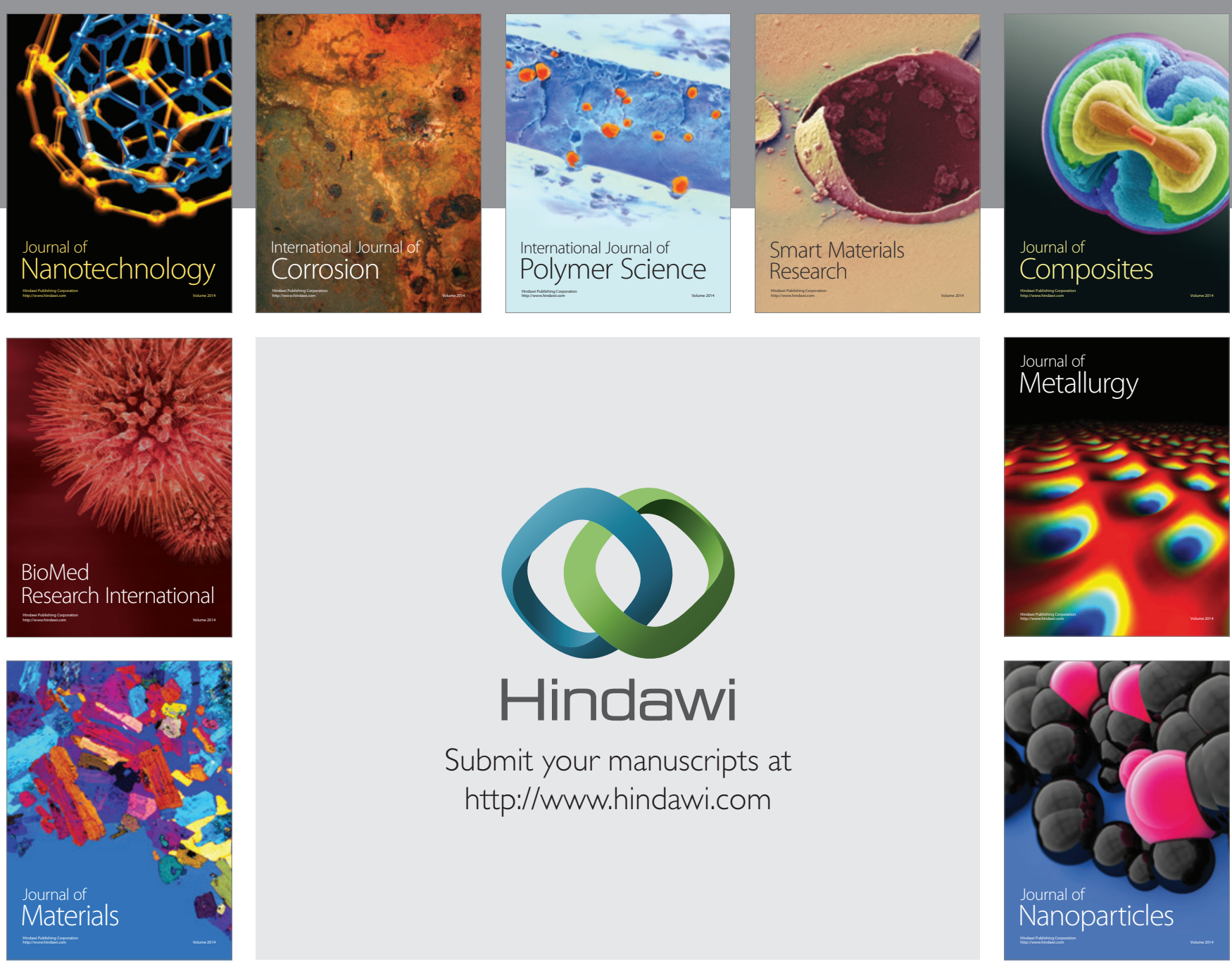

Submit your manuscripts at http://www.hindawi.com
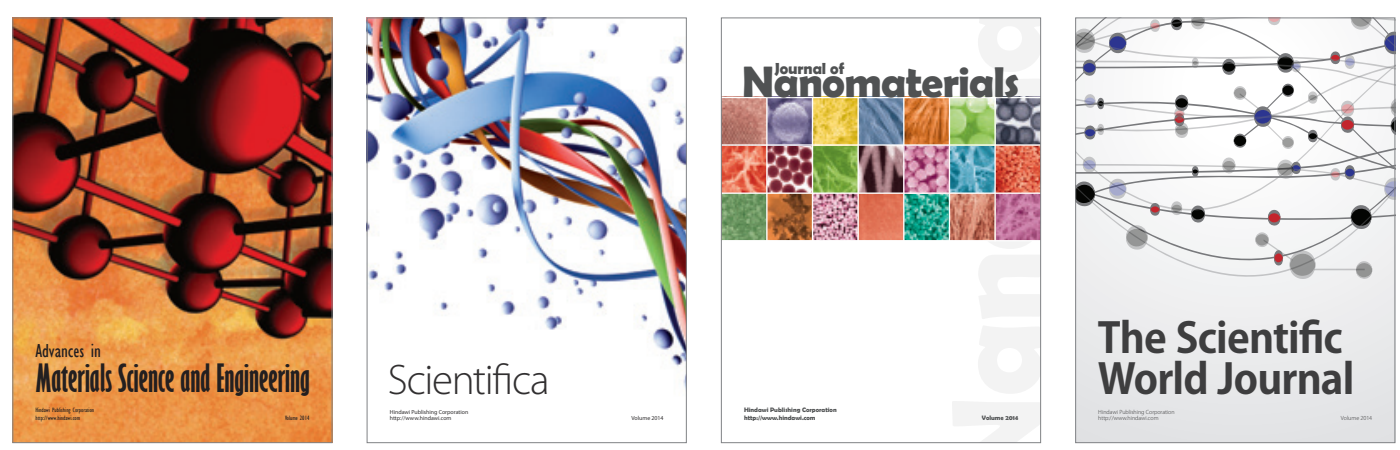

\section{The Scientific World Journal}
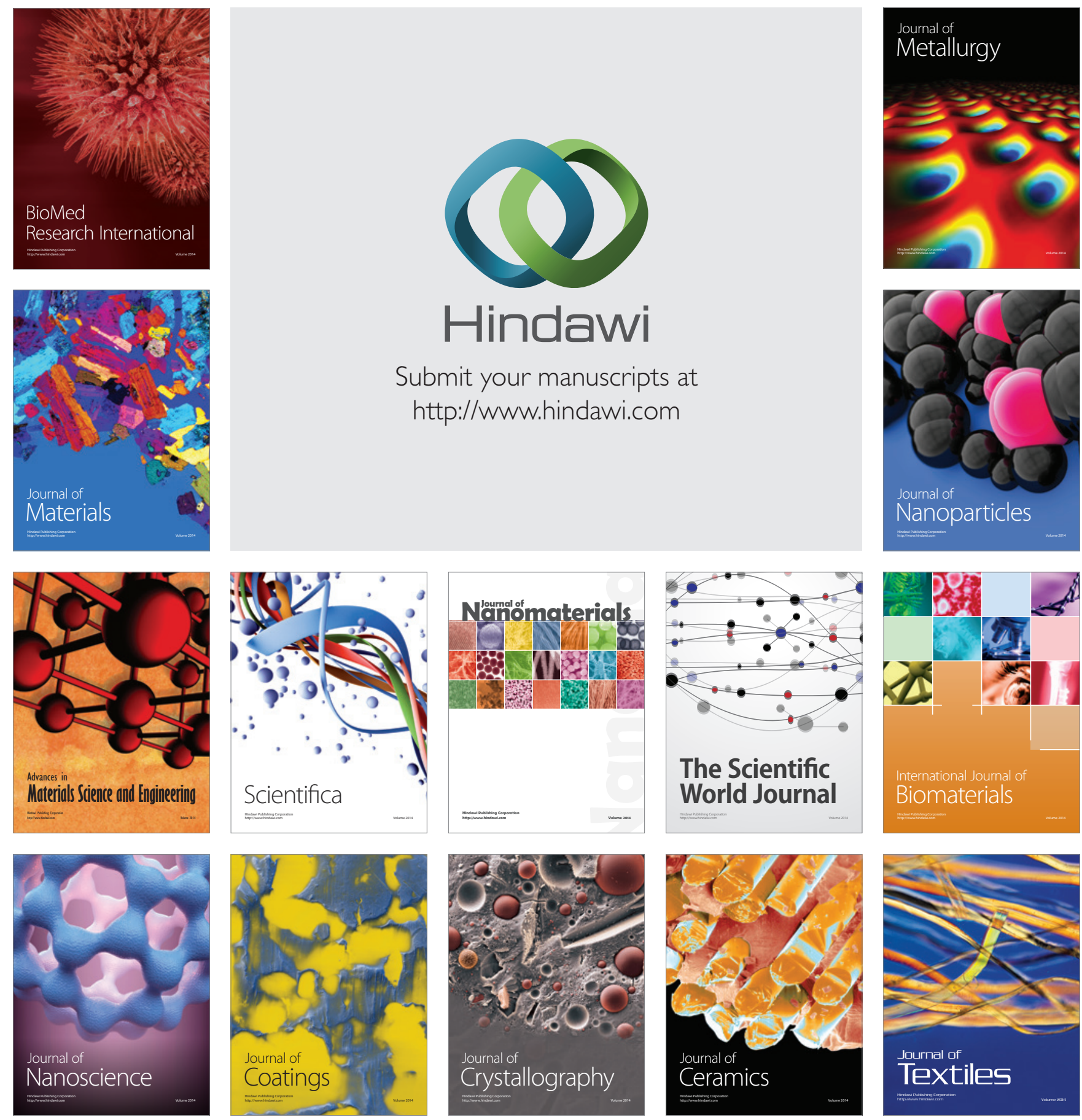